\title{
ENTROPY OF SYSTOLICALLY EXTREMAL SURFACES AND ASYMPTOTIC BOUNDS ${ }^{1}$
}

\author{
MIKHAIL G. KATZ* AND STÉPHANE SABOURAU
}

\begin{abstract}
We find an upper bound for the entropy of a systolically extremal surface, in terms of its systole. We combine the upper bound with A. Katok's lower bound in terms of the volume, to obtain a simpler alternative proof of M. Gromov's asymptotic estimate for the optimal systolic ratio of surfaces of large genus. Furthermore, we improve the multiplicative constant in Gromov's theorem. We show that every surface of genus at least 20 is Loewner. Finally, we relate, in higher dimension, the isoembolic ratio to the minimal entropy.
\end{abstract}

\section{Contents}

1. Entropv and svstole 1

2. The results 3

3. Basic estimate 4

4. Asvmptotic behavior of svstolic ratio for large genus 8

5. When is a surface Loewner? 9

6. Isoembolic ratio minimal entropv, and simplicial norm 10

$\begin{array}{ll}\text { References } & 12\end{array}$

\section{ENTROPY AND SYSTOLE}

We show that the volume entropy $h$ (together with A. Katok's optimal inequality for $h$ ) is the "right" intermediary in a transparent proof of M. Gromov's asymptotic bound for the systolic ratio of surfaces of large genus.

${ }^{1}$ Ergodic Theory and Dynamical Systems, to appear. See arXiv:math.DG/0410312

1991 Mathematics Subject Classification. Primary 53C20, 53C23 Secondary 28D20 .

Key words and phrases. isoembolic ratio, Katok's theorem, Loewner theorem, systole, volume entropy, minimal entropy.

*Supported by the Israel Science Foundation (grants no. 620/00-10.0 and 84/03). 
In this section, we review the definitions of minimal entropy and systole. The main results of this paper are presented in Section 2

Let $(M, \mathcal{G})$ be an $n$-dimensional closed Riemannian manifold. Denote by $(\tilde{M}, \tilde{\mathcal{G}})$ the universal Riemannian cover of $(M, \mathcal{G})$. Choose a point $\tilde{x}_{0} \in \tilde{M}$. The volume entropy (or asymptotic volume) $h(M, \mathcal{G})$ of $(M, \mathcal{G})$ is defined as follows:

$$
h(M, \mathcal{G})=\lim _{R \rightarrow+\infty} \frac{\log \left(\operatorname{vol}_{\tilde{\mathcal{G}}} B\left(\tilde{x}_{0}, R\right)\right)}{R},
$$

where $\operatorname{vol}_{\tilde{\mathcal{G}}} B\left(\tilde{x}_{0}, R\right)$ is the volume of the ball of radius $R$ centered at $\tilde{x}_{0} \in \tilde{M}$. Since $M$ is compact, the limit in (1.1) exists and does not depend on the point $\tilde{x}_{0} \in \tilde{M}$ Ma79. This asymptotic invariant describes the exponential growth rate of the volume in the universal cover.

Define the minimal volume entropy of $M$ as the infimum of the volume entropy of metrics of unit volume on $M$, or equivalently

$$
\operatorname{MinEnt}(M)=\inf _{\mathcal{G}} h(M, \mathcal{G}) \cdot \operatorname{vol}(M, \mathcal{G})^{\frac{1}{n}}
$$

where $\mathcal{G}$ runs over the space of all metrics on $M$.

The classical result of A. Katok Kato83 states that every metric $\mathcal{G}$ on a closed surface $M$ with negative Euler characteristic $\chi(M)$ satisfies the optimal inequality

$$
h(\mathcal{G})^{2} \geq \frac{2 \pi|\chi(M)|}{\operatorname{area}(\mathcal{G})} .
$$

Inequality (1.3) also holds for hom $\operatorname{ent}(\mathcal{G})$ Kato83, as well as the topological entropy, since the volume entropy bounds from below the topological entropy (see Ma79]).

The systole of a nonsimply connected closed Riemannian n-manifold $(M, \mathcal{G})$ is defined as

$$
\operatorname{sys} \pi_{1}(M, \mathcal{G})=\inf _{\gamma}\{\operatorname{length}(\gamma) \mid \gamma \text { a noncontractible loop of } M\} \text {. }
$$

We define the systolic ratio $\mathrm{SR}$ of $(M, \mathcal{G})$ as

$$
\operatorname{SR}(M, \mathcal{G})=\frac{\operatorname{sys} \pi_{1}(M, \mathcal{G})^{n}}{\operatorname{vol}(M, \mathcal{G})}
$$

and the optimal systolic ratio of $M$ as

$$
\operatorname{SR}(M)=\sup _{\mathcal{G}} \operatorname{SR}(M, \mathcal{G}),
$$

where $\mathcal{G}$ runs over the space of all metrics on $M$. 
C. Loewner proved the first systolic inequality. Namely, he showed that every metric $\mathcal{G}$ on the torus $\mathbb{T}^{2}$ satisfies the inequality

$$
\operatorname{sys}_{1}(\mathcal{G})^{2} \leq \frac{2}{\sqrt{3}} \operatorname{area}(\mathcal{G})
$$

with equality if and only if the metric $\mathcal{G}$ is flat, while the group of deck transformations of $\left(\mathbb{T}^{2}, \mathcal{G}\right)$ is a lattice homothetic to the lattice spanned by the cube roots of unity in $\mathbb{C}$. Thus, we have

$$
\operatorname{SR}\left(\mathbb{T}^{2}\right)=\frac{2}{\sqrt{3}}
$$

See [CK03] for a recent account on systolic inequalities. Asymptotic bounds for higher systoles are studied in Katz03. Systolic geometry has recently seen a period of rapid growth. The papers Ba03, Ba04, BB04 study the 1-systolic constant of higher-dimensional manifolds. Optimal generalisations of the Loewner inequality are studied in [Am04, BK03, BK04, BCIK04, IK04, KL04. A generalisation of $\mathrm{Pu}$ 's inequality is studied in BCIK05. A general framework for systolic geometry in a topological context is proposed in KR04. The most recent developments may be found in Sa04, KS04, KS05, Sa05, Sa06.

In Section 2, we present the main results of the paper. In Section 3 , we describe the basic estimate, based on a maximal packing argument combined with area lower bounds for systolically optimal surfaces. In Section 4. we combine the basic estimate with Katok's inequality to prove one of our main results, Theorem 2.2. In Section 5. we prove that every surface of genus at least 20 is Loewner. The last section contains higher dimensional generalisations and a proof of Theorem 2.3.

\section{The RESUlts}

We will relate the minimal entropy of a closed surface to its optimal systolic ratio. Namely, we find an upper bound for the entropy of a systolically extremal surface, in terms of its systole.

Proposition 2.1. Every extremal metric $\mathcal{G}$ on a surface $M$ satisfies

$$
h(\mathcal{G}) \leq-\frac{1}{\beta \operatorname{sys}_{1}(\mathcal{G})} \log \left(2 \alpha^{2} \operatorname{SR}(M)\right),
$$

whenever $\alpha, \beta>0$ and $4 \alpha+\beta<\frac{1}{2}$.

We combine this upper bound with A. Katok's optimal lower bound in terms of the volume, see (1.3), to obtain a simpler alternative proof of M. Gromov's asymptotic estimate for the optimal systolic ratio of surfaces of large genus. Furthermore, we improve the multiplicative constant in Gromov's theorem (see Section 4 for further details). 
Theorem 2.2. A surface $M_{g}$ of genus $g$ satisfies the bound

$$
\mathrm{SR}\left(M_{g}\right) \leq \frac{\log ^{2} g}{\pi g}(1+o(1)) \text { when } g \rightarrow \infty \text {. }
$$

An alternative approach is taken by F. Balacheff Bal04, but his constant is not as good as Gromov's, see Section 4. Furthermore, the approach of the present paper lends itself to higher-dimensional generalisations $\mathrm{Sa06}$.

As an application, we show that every metric on a surface of genus at least 20 satisfies the Loewner inequality (1.6) for the torus. This improves the best earlier estimate of 50 .

Finally, we relate, in higher dimension, the optimal isoembolic ratio to the minimal entropy (1.2). Recall that the optimal isoembolic ratio of an $n$-manifold $M$ is defined as

$$
\operatorname{Emb}(M)=\inf _{\mathcal{G}} \frac{\operatorname{vol}(M, \mathcal{G})}{\operatorname{inj}(M, \mathcal{G})^{n}}
$$

where $\mathcal{G}$ runs over the space of all metrics on $M$ and $\operatorname{inj}(M, \mathcal{G})$ is the injectivity radius of $(M, \mathcal{G})$. We show the following (see Section 6 for further details).

Theorem 2.3. There exits a positive constant $\lambda_{n}$ such that every $n$ manifold $M$ satisfies

$$
\operatorname{Emb}(M) \geq \lambda_{n} \frac{\operatorname{MinEnt}(M)^{n}}{\log ^{n}(1+\operatorname{MinEnt}(M))} .
$$

\section{BASIC ESTIMATE}

In Lemma 3.6 we recall the following well-known fact: the volume entropy agrees with the exponential growth rate of orbits under the action of the fundamental group in the universal cover, sometimes called the critical exponent.

We will also need the following estimate, $c f$. (3.5). M. Gromov showed in Gr83 that every aspherical closed surface $M$ satisfies the inequality

$$
\mathrm{SR}(M) \leq \frac{4}{3}
$$

He also showed that every nonsimply connected closed surface admits an extremal metric in a suitable generalized sense, namely a metric $\mathcal{G}_{\text {ex }}$ with optimal systolic ratio $\operatorname{SR}\left(M, \mathcal{G}_{\text {ex }}\right)=\mathrm{SR}(M)$. Furthermore, the disks $D(x, r)$ of radius $r \leq \frac{1}{2} \operatorname{sys} \pi_{1}\left(\mathcal{G}_{\text {ex }}\right)$ of extremal surfaces satisfy the bound

$$
\text { area } D(x, r) \geq 2 r^{2} \text {. }
$$


We will use arguments developed in Kato83, p. 357] to prove Proposition [3.1] below. Related arguments have been exploited in BM02, Ge04.

Proposition 3.1. Every extremal metric $\mathcal{G}$ on a surface $M$ satisfies

$$
h(\mathcal{G}) \leq-\frac{1}{\beta \operatorname{sys} \pi_{1}(\mathcal{G})} \log \left(2 \alpha^{2} \operatorname{SR}(M)\right),
$$

whenever $\alpha, \beta>0$ and $4 \alpha+\beta<\frac{1}{2}$.

Remark 3.2. As shown in Sa05], the volume entropy of surfaces with unit systole is bounded from above by a constant which does not depend on the metric. However, the constant found is not as good as in inequality (3.3).

Proof of Proposition 3.1. The idea is to bound from above the number of homotopy classes of based loops in $M$, by deforming such a loop into a fixed subgraph in $M$. The subgraph can be allowed to be as "coarse" as the size of the systole of $M$. More precisely, let $x_{0} \in M$ be a fixed basepoint. Consider a maximal system of disjoint disks

$$
D_{i}=D\left(x_{i}, R\right) \subset M
$$

of radius $R=\alpha \operatorname{sys} \pi_{1}(\mathcal{G})$ and centers $x_{i}$ with $i \in I$, including $x_{0}$. Since the metric $\mathcal{G}$ is assumed extremal, inequality (3.2) implies

$$
\text { area } D_{i} \geq 2 \alpha^{2} \operatorname{sys} \pi_{1}(\mathcal{G})^{2} \quad \forall i \in I \text {. }
$$

Therefore, this system admits at most $\frac{\operatorname{area}(\mathcal{G})}{2 \alpha^{2} \operatorname{sys} \pi_{1}(\mathcal{G})^{2}}$ disks. Thus,

$$
|I| \leq\left(2 \alpha^{2} \mathrm{SR}(M)\right)^{-1} \text {. }
$$

Let $c:[0, T] \rightarrow M$ be a geodesic loop of length $T$ based at $x_{0}$. Let

$$
m=\left[\frac{T}{\beta \operatorname{sys} \pi_{1}(\mathcal{G})}\right]
$$

be the integer part. The point $p_{0}=x_{0}$, together with the points

$$
p_{k}=c\left(k \beta \operatorname{sys}_{1}(\mathcal{G})\right), \quad k=1, \ldots, m
$$

and the point $p_{m+1}=x_{0}$, partition the loop $c$ into $m+1$ segments of length at most $\beta$ sys $\pi_{1}(\mathcal{G})$. Since the system of disks $D_{i}$ is maximal, the disks of radius $2 R=2 \alpha \operatorname{sys} \pi_{1}(\mathcal{G})$ centered at $x_{i}$ cover $M$. Therefore, for every $p_{k}$, a nearest point $q_{k}$ among the centers $x_{i}$, is at distance at most $2 R$ from $p_{k}$. Consider the loop

$$
\alpha_{k}=c_{k} \cup\left[p_{k+1}, q_{k+1}\right] \cup\left[q_{k+1}, q_{k}\right] \cup\left[q_{k}, p_{k}\right],
$$


where $c_{k}$ is the arc of $c$ joining $p_{k}$ to $p_{k+1}$, while $[x, y]$ denotes a minimizing path joining $x$ to $y$. Then

$$
\text { length }\left(\alpha_{k}\right) \leq 2(4 \alpha+\beta) \operatorname{sys}_{1}(\mathcal{G})<\operatorname{sys}_{1}(\mathcal{G}),
$$

by our hypothesis on $\alpha, \beta$. Thus the loop $\alpha_{k}$ is contractible. The same is true for the loops $c_{0} \cup\left[p_{1}, q_{1}\right] \cup\left[q_{1}, x_{0}\right]$ and $c_{m} \cup\left[x_{m}, q_{m}\right] \cup\left[q_{m}, p_{m}\right]$.

Therefore, the geodesic loop $c$ is homotopic to a piecewise geodesic loop

$$
c^{\prime}=\left(x_{0}, q_{1}, \ldots, q_{m}, x_{0}\right)
$$

Note that the minimizing path from $p_{k}$ to $q_{k}$ may not be unique, but we choose one, being careful that the same choice is used on "both sides", i.e. for both loops $\alpha_{k-1}$ and $\alpha_{k}$.

Thus, two nonhomotopic closed geodesic loops $c_{1}$ and $c_{2}$ based at $x_{0}$, give rise to two distinct loops $c_{1}^{\prime}$ and $c_{2}^{\prime}$ as in (3.8). In constructing the loops $c^{\prime}$, we always choose the same minimizing path between a given pair of points $q_{k}$. Thus, the number $P^{\prime}(T)$ of homotopy classes which can be represented by loops of length $T$ based at $x_{0}$ satisfies

$$
\begin{aligned}
P^{\prime}(T) & \leq|I|^{m} \\
& \leq|I|^{\frac{T}{\beta \mathrm{sys} \pi 1(\mathcal{G})}} \\
& \leq\left(2 \alpha^{2} \mathrm{SR}(M)\right)^{-\frac{T}{\beta \mathrm{sys} \pi_{1}(\mathcal{G})}},
\end{aligned}
$$

and the proposition now follows from Lemma 3.6

Remark 3.3. Instead of relying on the existence of systolically extremal surfaces, we could have exploited instead $\epsilon$-regular surfaces satisfying (3.2) for $r \geq \epsilon$, whose existence is considerably easier to establish [Gr83, 5.6.C"]. Since the choice of $\alpha$ in (4.5) entails exploiting packings by arbitrarily small disks in (3.4), we have to be careful to choose $\epsilon<\alpha$ sys $\pi_{1}(M)$.

Remark 3.4. A similar technique can be used to bound the number of free homotopy classes of loops in $X$. In higher dimensions, the latter bound seems to be less useful, therefore we chose to bound the number of based loops.

Combining inequalities (1.3) and (3.3), we obtain the following corollary.

Corollary 3.5. Let $M_{g}$ be a closed orientable surface of genus $g$. Whenever $4 \alpha+\beta<\frac{1}{2}$, we have

$$
\frac{\log ^{2}\left(2 \alpha^{2} \operatorname{SR}\left(M_{g}\right)\right)}{\operatorname{SR}\left(M_{g}\right)} \geq 4 \pi \beta^{2}(g-1) \text {. }
$$


We conclude this section by recalling the following well-known fact, cf. [KH95, Proposition 9.6.6, p. 374].

Lemma 3.6. Let $(M, \mathcal{G})$ be a closed Riemannian manifold. Then,

$$
h(M, \mathcal{G})=\lim _{T \rightarrow+\infty} \frac{\log \left(P^{\prime}(T)\right)}{T}
$$

where $P^{\prime}(T)$ is the number of homotopy classes of loops based at some fixed point $x_{0}$ which can be represented by loops of length at most $T$.

Proof. Fix $x_{0}$ in $M$ and a lift $\tilde{x}_{0}$ in the universal cover $\tilde{M}$. The group

$$
\Gamma:=\pi_{1}\left(M, x_{0}\right)
$$

acts on $\tilde{M}$ by isometries. The orbit of $\tilde{x}_{0}$ by $\Gamma$ is denoted $\Gamma . \tilde{x}_{0}$. Consider a fundamental domain $\Delta$ for the action of $\Gamma$, containing $\tilde{x}_{0}$. Denote by $D$ the diameter of $\Delta$. The cardinal of $\Gamma . \tilde{x}_{0} \cap B\left(\tilde{x}_{0}, R\right)$ is bounded from above by the number of translated fundamental domains $\gamma \cdot \Delta$, where $\gamma \in \Gamma$, contained in $B\left(\tilde{x}_{0}, R+D\right)$. It is also bounded from below by the number of translated fundamental domains $\gamma \cdot \Delta$ contained in $B\left(\tilde{x}_{0}, R\right)$. Therefore, we have

$$
\frac{\operatorname{vol}\left(B\left(\tilde{x}_{0}, R\right)\right)}{\operatorname{vol}(M, \mathcal{G})} \leq \operatorname{card}\left(\Gamma . \tilde{x}_{0} \cap B\left(\tilde{x}_{0}, R\right)\right) \leq \frac{\operatorname{vol}\left(B\left(\tilde{x}_{0}, R+D\right)\right)}{\operatorname{vol}(M, \mathcal{G})}
$$

Take the log of these terms and divide by $R$. The lower bound becomes

$$
\begin{aligned}
\frac{1}{R} & \log \left(\frac{\operatorname{vol}(B(R))}{\operatorname{vol}(\mathcal{G})}\right)= \\
& =\frac{1}{R} \log (\operatorname{vol}(B(R)))-\frac{1}{R} \log (\operatorname{vol}(\mathcal{G})),
\end{aligned}
$$

and the upper bound becomes

$$
\begin{aligned}
\frac{1}{R} & \log \left(\frac{\operatorname{vol}(B(R+D))}{\operatorname{vol}(\mathcal{G})}\right)= \\
& =\frac{R+D}{R} \frac{1}{R+D} \log (\operatorname{vol}(B(R+D)))-\frac{1}{R} \log (\operatorname{vol}(\mathcal{G})) .
\end{aligned}
$$

Hence both bounds tend to $h(\mathcal{G})$ when $R$ goes to infinity. Therefore,

$$
h(\mathcal{G})=\lim _{R \rightarrow+\infty} \frac{1}{R} \log \left(\operatorname{card}\left(\Gamma . \tilde{x}_{0} \cap B\left(\tilde{x}_{0}, R\right)\right)\right) .
$$

This yields the result since $P^{\prime}(R)=\operatorname{card}\left(\Gamma . \tilde{x}_{0} \cap B\left(\tilde{x}_{0}, R\right)\right)$. 


\section{Asymptotic Behavior of Systolic Ratio For LARGe Genus}

We now consider the asymptotic behavior of the optimal systolic ratio of surfaces. M. Gromov [Gr83, p. 74] established a bound for the ratio $\operatorname{SR}\left(M_{g}\right)$ by using a technique known as "diffusion of chains". The multiplicative constant $\frac{1}{\pi}$ in (4.2) below improves the constant 4 which could be obtained from the techniques in Gr83. F. Balacheff Bal04 found another proof of a similar inequality, by combining the works of S. Kodani [Ko87], and B. Bollobás and E. Szemerédi [BoS02] on systolic inequalities of graphs, obtaining a multiplicative constant of $\frac{8}{3(\log 2)^{2}}$.

Theorem 4.1. Given a real number $\lambda<\pi$, every surface $M_{g}$ of genus $g$ satisfies

$$
\mathrm{SR}\left(M_{g}\right) \leq \frac{\log ^{2} g}{\lambda g}
$$

for g large enough. Thus,

$$
\mathrm{SR}\left(M_{g}\right) \lesssim \frac{\log ^{2} g}{\pi g} \quad \text { as } g \rightarrow \infty .
$$

Remark 4.2. A lower bound for $\mathrm{SR}\left(M_{g}\right)$ was found by P. Buser and P. Sarnak BS94. Namely, they construct hyperbolic surfaces $\left(M_{g}, \mathcal{G}_{g}\right)$ of arbitrarily large genus $g$ obtained as congruence coverings of an arithmetic Riemann surface, such that

$$
\mathrm{SR}\left(M_{g}, \mathcal{G}_{g}\right) \gtrsim \frac{4}{9 \pi} \frac{\log ^{2} g}{g} .
$$

Therefore, we have

$$
\frac{4}{9 \pi} \leq \limsup _{g \rightarrow \infty} \operatorname{SR}\left(M_{g}\right) \frac{g}{\log ^{2} g} \leq \frac{1}{\pi} .
$$

Proof of Theorem 4.1. Let $\mathcal{G}$ be an extremal metric on $M_{g}$. We now apply Corollary 3.5. Note that $\operatorname{SR}\left(M_{g}, \mathcal{G}\right)$ tends to zero as the genus $g$ becomes unbounded, $c f$. (5.4). For a right choice of $\alpha$ and $\beta$, inequality (3.10) leads to the asymptotic implicit upper bound (4.6) on $\operatorname{SR}\left(M_{g}\right)$ below. Indeed, given $\lambda<\lambda_{+}<\pi$, we set

$$
\beta=\sqrt{\frac{\lambda_{+}}{4 \pi}}<\frac{1}{2},
$$

and choose

$$
\alpha<\frac{1}{4}\left(\frac{1}{2}-\beta\right) .
$$


Inequality (3.10) implies

$$
\frac{\log ^{2}\left(\operatorname{SR}\left(M_{g}\right)\right)}{\operatorname{SR}\left(M_{g}\right)} \geq \lambda g
$$

if $g$ is large enough. Now we want to invert this relation in order to get an asymptotic upper bound on $\operatorname{SR}\left(M_{g}\right)$. Let $\rho=\operatorname{SR}\left(M_{g}\right)^{-\frac{1}{2}}$ and $\delta=\frac{1}{2} \sqrt{\lambda g}$. Then inequality (4.6) yields the following estimate:

$$
\begin{aligned}
\rho \log \rho & \geq \delta \\
& \geq \delta-\frac{\delta \log \log \delta}{\log \delta} \\
& =\frac{\delta}{\log \delta} \log \left(\frac{\delta}{\log \delta}\right) .
\end{aligned}
$$

Since the function $x \log x$ is increasing for $x$ large enough, we deduce that $\rho \geq \frac{\delta}{\log \delta}$, and the latter inequality translates back into (4.1).

\section{WHEN IS A SURFACE LOEWNER?}

We now extend the classical Loewner inequality (1.6) on the torus to surfaces of higher genus. We will say that a surface $M$ is Loewner if $\operatorname{SR}(M) \leq \frac{2}{\sqrt{3}}$.

Theorem 5.1. Every surface of genus at least 20 is Loewner.

Proof. Let $\mathcal{G}$ be an extremal metric on $M$. If $\beta=\frac{1}{2}-4 \alpha$, inequality (3.10) yields

$$
\frac{\log ^{2}\left(2 \alpha^{2} \operatorname{SR}(M)\right)}{\operatorname{SR}(M)} \geq 4 \pi\left(\frac{1}{2}-4 \alpha\right)^{2}(g-1)
$$

for every $\alpha \leq \frac{1}{8}$.

Suppose now that $\operatorname{SR}(M)>\frac{2}{\sqrt{3}}$. Since $\operatorname{SR}(M) \leq \frac{4}{3}$ by (3.1), we have $2 \alpha^{2} \operatorname{SR}(M) \leq 1$ for every $\alpha \leq \frac{1}{8}$. Therefore,

$$
\frac{\sqrt{3}}{2} \log ^{2}\left(\frac{\sqrt{3}}{4 \alpha^{2}}\right) \geq 4 \pi\left(\frac{1}{2}-4 \alpha\right)^{2}(g-1)
$$

for every $\alpha \leq \frac{1}{8}$. Hence,

$$
\min _{0<\alpha \leq \frac{1}{8}} \frac{\sqrt{3}}{8 \pi}\left(\frac{\log \left(\frac{\sqrt{3}}{4 \alpha^{2}}\right)}{\frac{1}{2}-4 \alpha}\right)^{2} \geq g-1
$$

For $\alpha=.031$, the expression to minimize is about 18.201. Thus, $g \leq 19$. Therefore, every surface of genus greater or equal to 20 is Loewner. 
Note that M. Gromov Gr83, p. 50] (cf. [Ko87, Theorem 4, part (1)]) proved a general estimate which implies that

$$
\operatorname{SR}\left(M_{g}\right)<\frac{64}{4 \sqrt{g}+27}
$$

It follows from Gromov's estimate (5.4) that orientable surfaces satisfy Loewner inequality (1.6) if the genus $g$ is bigger than 50. Our theorem brings this bound down to 20. It has recently been shown KS04 that the genus 2 surface is Loewner. The remaining open cases are therefore $g=3, \ldots, 19$.

Remark 5.2. Let $\alpha=\frac{1}{30}$. Instead of taking an arbitrary packing, we start with a systolic loop, and choose 15 disjoint disks centered at equally spaced points of the loop. By an averaging procedure Gr83], we can get the combined area of these disks to be, not $15\left(2 r^{2}\right)$, but rather $15\left(3 r^{2}\right)$. Now we complete these 15 disks to a maximal packing, and argue as before. The only difference is that we have a better lower bound for the area of the disks.

If $\alpha=\frac{1}{30}$, then there are at most 382 balls in the packing of an unloewner surface. Hence the area of the packing is at least $2.039 \alpha^{2}$, instead of $2 \alpha^{2}$. Calculating the resulting expression in (5.3) yields about 18.12, which is better than 18.20 but not enough to dip under 18 .

\section{IsOEMBOLIC RATIO, MINIMAL ENTROPY, AND SIMPLICIAL NORM}

Analogous estimates can be proved in higher dimension. However, the results we obtain are weaker than in the previous sections, in the absence of similar results on the existence of systolically extremal metrics.

M. Berger proved in [Be80 that the isoembolic ratio $\operatorname{Emb}(M, \mathcal{G})$ of every Riemannian $n$-manifold $(M, \mathcal{G})$, defined as

$$
\operatorname{Emb}(M, \mathcal{G})=\frac{\operatorname{vol}(M, \mathcal{G})}{\operatorname{inj}(M, \mathcal{G})^{n}}
$$

satisfies

$$
\operatorname{Emb}(M, \mathcal{G}) \geq \operatorname{Emb}\left(S^{n}, \text { can }\right)
$$

with equality if and only if $M$ is a sphere, while $\mathcal{G}$ has constant sectional curvature. In particular, $\operatorname{Emb}(M) \geq \operatorname{Emb}\left(S^{n}\right)$, where Emb is the optimal isoembolic ratio defined in (2.3).

C. Croke showed in $\mathrm{Cr} 80$ that for every Riemannian manifold $(M, \mathcal{G})$,

$$
\operatorname{vol} B(R) \geq c_{n} R^{n}
$$


for every $R \leq \frac{1}{2} \operatorname{inj}(\mathcal{G})$, where $\operatorname{vol} B(R)$ is the volume of a ball of radius $R$ in $M$. Furthermore, he showed in $\mathrm{Cr} 88$, that

$$
\operatorname{Emb}(M) \geq C_{n} \operatorname{ballcat}(M),
$$

where ballcat $(M)$ is the minimum number $n$ such that $M$ can be covered by $n+1$ topological balls. If $M$ is a closed $n$-dimensional manifolds, then ballcat $(M) \leq n$ (see CT03, p. 77]). Note also that T. Yamaguchi Ya88 proved that, for every real $C$, the class of closed $n$-dimensional manifolds $M$ satisfying $\operatorname{Emb}(M) \leq C$ contains finitely many homotopy types. Furthermore, I. Babenko [Ba93, Theorem 1.2, p. 6] showed that the minimal volume entropy is a homotopy invariant of $M$.

The following analogue of Proposition 3.1] holds.

Proposition 6.1. Every Riemannian n-manifold $(M, \mathcal{G})$ satisfies

$$
h(\mathcal{G}) \leq \frac{1}{\beta \operatorname{inj}(\mathcal{G})} \log \left(\frac{\operatorname{Emb}(\mathcal{G})}{c_{n} \alpha^{n}}\right)
$$

whenever $\alpha, \beta>0$ and $4 \alpha+\beta<\frac{1}{2}$.

The proof is identical to that of Proposition 3.1, with $c_{n}$ from (6.3) replacing the coefficient 2 in (3.5), (3.6) and (3.9), and $n$ replacing the dimension 2.

Arguing as in Theorem 4.1, we obtain the following theorem (compare with (6.4)).

Theorem 6.2. There exits a positive constant $\lambda_{n}$ such that every $n$ manifold $M$ satisfies

$$
\operatorname{Emb}(M) \geq \lambda_{n} \frac{\operatorname{MinEnt}(M)^{n}}{\log ^{n}(1+\operatorname{MinEnt}(M))}
$$

G. Besson, G. Courtois, and S. Gallot BCG95 proved that the minimal entropy of a closed negatively curved locally symmetric $n$ manifold $\left(M, \mathcal{G}_{0}\right)$ satisfies

$$
\operatorname{MinEnt}(M)^{n}=\operatorname{vol}\left(\mathcal{G}_{0}\right) \cdot h\left(\mathcal{G}_{0}\right)^{n} .
$$

This theorem generalizes (1.3).

Remark 6.3. The relation (6.7) is a sharp version, for negatively curved locally symmetric manifolds, of the following general result of M. Gromov (see [Gr81, p. 37]). Every $n$-manifold $M$ with simplicial volume $\|M\|$ satisfies

$$
\operatorname{MinEnt}(M)^{n} \geq C_{n}\|M\| \text {. }
$$


Therefore, inequalities (6.6) and (6.8) show that there exists a positive constant $\lambda_{n}^{\prime}$ such that

$$
\operatorname{Emb}(M) \geq \lambda_{n}^{\prime} \frac{\|M\|}{\log ^{n}(1+\|M\|)}
$$

M. Gromov proved in Gr83, p. 74] that there exists a positive constant $\lambda_{n}^{\prime \prime}$ such that

$$
\mathrm{SR}(M) \leq \lambda_{n}^{\prime \prime} \frac{\log ^{n}(1+\|M\|)}{\|M\|} .
$$

Thus, inequality (6.9) appears as a particular case of (6.10).

Question 6.4. Are there manifolds with large minimal entropy and small simplicial volume? Such manifolds (if they exist) would provide examples where (6.6) yields a better estimate than (6.10).

More generally, in the presence of a lower bound for volumes of balls, our argument yields the following result.

Proposition 6.5. Let $\mathcal{G}$ be a metric on a closed $n$-manifold $M$ such that for some $c>0$, every ball of radius $r$ with $0<r<\frac{1}{2} \operatorname{sys}_{1}(\mathcal{G})$ satisfies

$$
\text { area } B(r) \geq c r^{n} \text {. }
$$

Then, we have

$$
h(\mathcal{G}) \leq-\frac{1}{\beta \operatorname{sys}_{1}(\mathcal{G})} \log \left(c \alpha^{n} \operatorname{SR}(M, \mathcal{G})\right),
$$

whenever $\alpha, \beta>0$ and $4 \alpha+\beta<\frac{1}{2}$. Therefore, there exists a positive constant $\lambda=\lambda(n, c)$ such that

$$
\operatorname{SR}(M, \mathcal{G}) \geq \lambda(n, c) \frac{\operatorname{MinEnt}(M)^{n}}{\log ^{n}(1+\operatorname{MinEnt}(M))} .
$$

\section{REFERENCES}

[Am04] Ammann, B.: Dirac eigenvalue estimates on two-tori. J. Geom. Phys. 51 (2004), no. 3, 372-386.

[Ba93] Babenko, I.: Asymptotic invariants of smooth manifolds. Russian Acad. Sci. Izv. Math. 41 (1993), 1-38.

[Ba03] Babenko, I.: Nature topologique des systoles unidimensionnelles, preprint 03-07 (2003). Available at the site http://www.math.univ-montp2.fr/lsprepub/prepub.html

[Ba04] Babenko, I.: Géométrie systolique des variétés de groupe fondamental $\mathbb{Z}_{2}$, Sémin. Théor. Spectr. Géom. Grenoble, 22 (2004), 25-52.

[BB04] Babenko, I.; Balacheff, F.: Géométrie systolique des sommes connexes et des revêtements cycliques, preprint. 
[Bal04] Balacheff, F.: Sur des problèmes de la géométrie systolique. Sémin. Théor. Spectr. Géom. Grenoble, 22 (2004), 71-82.

[BCIK04] Bangert, V; Croke, C.; Ivanov, S.; Katz, M.: Boundary case of equality in optimal Loewner-type inequalities, Trans. Amer. Math. Soc., to appear. See arXiv:math.DG/0406008

[BCIK05] Bangert, V; Croke, C.; Ivanov, S.; Katz, M.: Filling area conjecture and ovalless real hyperelliptic surfaces, Geometric and Functional Analysis (GAFA), to appear. See arXiv:math.DG/0405583

[BK03] Bangert, V.; Katz, M.: Stable systolic inequalities and cohomology products, Comm. Pure Appl. Math. 56 (2003), 979-997. Available at arXiv:math.DG/0204181

[BK04] Bangert, V; Katz, M.: An optimal Loewner-type systolic inequality and harmonic one-forms of constant norm. Comm. Anal. Geom. 12 (2004), number 3, 703-732. See arXiv: math.DG/0304494

[Be80] Berger, M.: Une borne inférieure pour le volume d'une variété riemannienne en fonction du rayon d'injectivité. Ann. Inst. Fourier 30 (1980), 259-265.

[BCG95] Besson, G.; Courtois, G.; Gallot, S.: Volume et entropie minimale des espaces localement symétriques. Invent. Math. 103 (1991), 417-445.

[BoS02] Bollobás, B.; Szemerédi, E.: Girth of sparse graphs. J. Graph Theory 39 (2002), 194-200.

[BM02] Burger, M.; Gelander, T.; Lubotzky, A.; Mozes, S.: Counting hyperbolic manifolds. Geom. Funct. Anal. 12 (2002), no. 6, 1161-1173.

[BS94] Buser, P.; Sarnak, P.: On the period matrix of a Riemann surface of large genus. With an appendix by J. H. Conway and N. J. A. Sloane. Invent. Math. 117 (1994), no. 1, 27-56.

[CT03] Cornea, O.; Lupton, G.; Oprea, J.; Tanré, D.: Lusternik-Schnirelmann category. Mathematical Surveys and Monographs, 103. American Mathematical Society, Providence, RI, 2003.

[Cr80] Croke, C.: Some isoperimetric inequalities and eigenvalue estimates. Ann. Sci. École Norm. Sup. 13 (1980), 519-535.

[Cr88] Croke, C.: An isoembolic pinching theorem. Invent. Math. 92 (1988), $385-387$.

[CK03] Croke, C.; Katz, M.: Universal volume bounds in Riemannian manifolds, Surveys in Differential Geometry VIII, Lectures on Geometry and Topology held in honor of Calabi, Lawson, Siu, and Uhlenbeck at Harvard University, May 3- 5, 2002, edited by S.T. Yau (Somerville, MA: International Press, 2003.) pp. 109 - 137. See arXiv:math.DG/0302248

[Ge04] Gelander, T.: Homotopy type and volume of locally symmetric manifolds. Duke Math. J. 124 (2004), no. 3, 459-515.

[Gr81] Gromov, M.: Volume and bounded cohomology. Publ. IHES. 56 (1981), 213-307.

[Gr83] Gromov, M.: Filling Riemannian manifolds. J. Differential Geom. 18 (1983), 1-147.

[Gr96] Gromov, M.: Systoles and intersystolic inequalities. In: Actes de la Table Ronde de Géométrie Différentielle (Luminy 1992), 291-362. Séminaires et Congrès, 1. Soc. Math. France, Institut Henri Poincaré, Paris 1996. 
[Gr99] Gromov, M.: Metric structures for Riemannian and non-Riemannian spaces. Progress in Mathematics 152. Birkhäuser, Boston 1999.

[IK04] Ivanov, S.; Katz, M.: Generalized degree and optimal Loewner-type inequalities, Israel J. Math. 141 (2004), 221-233. arXiv:math.DG/0405019

[Kato83] Katok, A.: Entropy and closed geodesics, Ergo. Th. Dynam. Sys. 2 (1983), 339-365.

[KH95] Katok, A.; Hasselblatt, B.: Introduction to the modern theory of dynamical systems. With a supplementary chapter by Katok and Leonardo Mendoza. Encyclopedia of Mathematics and its Applications, 54. Cambridge University Press, Cambridge, 1995.

[Katz03] Katz, M.: Four-manifold systoles and surjectivity of period map, Comment. Math. Helv. 78 (2003), 772-786. arXiv:math.DG/0302306

[KL04] Katz, M.; Lescop, C.: Filling area conjecture, optimal systolic inequalities, and the fiber class in abelian covers, Proceedings of conference and workshop in memory of R. Brooks, held at the Technion, Israel Mathematical Conference Proceedings, Contemporary Math., Amer. Math. Soc., Providence, R.I. (to appear). See arXiv:math.DG/0412011

[KR04] Katz, M.; Rudyak, Y.: Lusternik-Schnirelmann category and systolic category of low dimensional manifolds. Communications on Pure and Applied Mathematics, to appear. See arXiv:math.DG/0410456

[KS04] Katz, M.; Sabourau, S.: Hyperelliptic surfaces are Loewner, Proc. Amer. Math. Soc. (to appear). See arXiv:math.DG/0407009

[KS05] Katz, M.; Sabourau, S.: An optimal systolic inequality for CAT(0) metrics in genus two, preprint.

[Ko87] Kodani, S.: On two-dimensional isosystolic inequalities, Kodai Math. J. 10 (1987) no. 3, 314-327.

[Ma79] Manning, A.: Topological entropy for geodesic flows, Ann. of Math. (2) 110 (1979), 567-573.

[Sa04] Sabourau, S.: Systoles des surfaces plates singulières de genre deux, Math. Zeitschrift 247 (2004), no. 4, 693-709.

[Sa05] Sabourau, S.: Entropy and systoles on surfaces, preprint.

[Sa06] Sabourau, S.: Systolic volume and minimal entropy of aspherical manifolds, preprint.

[Ya88] Yamaguchi, T.: Homotopy type finiteness theorems for certain precompact families of Riemannian manifolds, Proc. Amer. Math. Soc. 102 (1988), 660-666.

Department of Mathematics and Statistics, Bar Ilan University, RAMAT GAN 52900 ISRAEL

E-mail address: katzmik@math.biu.ac.il

Laboratoire de Mathématiques et Physique Théorique, Université de Tours, Parc de Grandmont, 37400 Tours, France

Mathematics and Computer Science Department, Saint-Joseph's University, 5600 City Avenue, Philadelphia, PA 19131, USA

E-mail address: sabourau@gargan.math.univ-tours.fr 